\title{
Classification of Atrial Tachyarrhythmias in Electrocardiograms Using Time Frequency Analysis
}

\author{
MG Tsipouras $^{1,2}$, VP Oikonomou ${ }^{1,2}$, DI Fotiadis ${ }^{1,2,3}$, LK Michalis ${ }^{3,4}$, D Sideris L $^{3,4}$ \\ ${ }^{1}$ Unit of Medical Technology and Intelligent Information Systems, Department of Computer Science, \\ University of Ioannina, GR 45110 Ioannina, Greece \\ ${ }^{2}$ Biomedical Research Institute - FORTH, GR 45110 Ioannina, Greece \\ ${ }^{3}$ Michaelidion Cardiology Center, GR 45110 Ioannina, Greece \\ ${ }^{4}$ Division of Cardiology, Medical School, GR 45110 Ioannina, Greece
}

\begin{abstract}
In this work a novel automated method, combining time-frequency analysis and expert's knowledge, for the classification of atrial tachyarrhythmias is presented. It is based on the analysis of small ECG segments and their classification into three categories of cardiac rhythm: (a) atrial fibrillation, (b) atrial flutter and (c) normal sinus rhythms. Time-frequency analysis is used to calculate the power spectrum density for each segment. Several spectral characteristics are extracted from the power spectrum density, representing the energy distribution on the time-frequency plane. These characteristics are used as input in an artificial neural network, which classifies each ECG segment into one of the three categories. The method is validated using the MIT-BIH arrhythmia database and the obtained average sensitivity and specificity are $93.4 \%$ and $96.5 \%$, respectively.
\end{abstract}

\section{Introduction}

The automated diagnosis of atrial tachyarrhythmias is a vital problem in clinical cardiology, especially when it is performed in real time. Several researchers have addressed the problem of automatic detection and classification of atrial tachyarrhythmias [1-3]. However, most of the approaches proposed use the entire ECG signal extracting several features from it, such as the $\mathrm{P}$ wave, which is an extremely time-consuming process and sometimes difficult due to the presence of noise. In addition the identification of normal sinus rhythm (NSR), atrial fibrillation (AFIB) and atrial flutter (AFL) based on Fourier analysis is not effective since ventricular activity overlaps with atrial activity in the frequency domain. The main difference between AFIB, AFL and NSR is the atrial activation of the heart.

This work focuses on the study of the ECG segments where the atrial activation of the heart is more distinctive.
An automated method for the classification of atrial tachyarrhythmias, is proposed based on time-frequency analysis. The approach is based on the analysis of small ECG segments and their classification into three categories of cardiac rhythms: (a) atrial fibrillation, (b) atrial flutter and (c) normal sinus rhythm. Time-frequency analysis is used to calculate the power spectrum density (PSD) for each segment. Several spectral characteristics are extracted from the PSD, representing the energy distribution on the time-frequency plane in certain areas of interest. These features are used as input in an artificial neural network (ANN), which classifies each ECG segment into one of the three categories. Recordings from the MIT-BIH arrhythmia database [4] and the MIT-BIH atrial fibrillation database [5] are used for training and evaluation of the method.

\section{Methods}

The proposed method consists of three steps: (a) preprocessing, which includes QRS detection and ECG segment creation; (b) time-frequency analysis and feature extraction and (c) classification of atrial tachyarrhythmias based on artificial neural network (ANN).

\subsection{Dataset}

The dataset used for the method includes 100.000 beats, selected from AFIB and AFL episodes and NSR annotated beats. The type of beats of the dataset are shown in Table 2: 59.500 normal annotated beats were randomly selected from the MIT-BIH arrhythmia database, 40.000 beats belonging to AFIB episodes from the MIT-BIH arrhythmia database and the MIT-BIH atrial fibrillation database and 500 beats belonging to AFL episodes from the MIT-BIH arrhythmia database. The number of beats belonging to the AFL category is much smaller due to the small number of AFL episodes existing in the database. 
Table 1. Dataset: number of beats for each type of cardiac rhythm.

\begin{tabular}{ll}
\hline Cardiac rhythm & $\mathrm{N}$ \\
\hline Normal & 59,500 \\
Atrial Fibrillation & 40,000 \\
Atrial Flutter & 500 \\
\hline Total & 100,000 \\
\hline
\end{tabular}

\subsection{Preprocessing}

Initially, the QRS complexes are detected and the start and end points for each QRS complex are determined. A modification of the algorithm proposed by Tompkins [6] is used for QRS detection. The algorithm includes two stages: (a) preprocessing of the ECG signal and (b) decision if a QRS complex is detected. During the preprocessing digital filters are used for the removal of the electromagnetic noise and the correction of the baseline wandering. Also features of the ECG that are not necessary for QRS detection, such as P and T waves, are removed. Substantially, a bandpass filter (combining a lowpass and a hightpass filter) with cut-off frequencies 5 and $17 \mathrm{~Hz}$ is applied, since in this range of frequencies the QRS energy is concentrated. After the filtering a differentiator and an integrator are applied. In the decision stage the candidate $\mathrm{R}$ peaks are extracted and rules are used to determine if a candidate peak is a QRS complex or not [6].

Other features, which are extracted, are the QRS start and end points. For this purpose a modification of the method described by Daskalov [7] is used. The method is carried out in two stages: (a) identification of two search intervals, one for the detection of the QRS start and the second for the detection of the QRS end and (b) determination of the QRS limits. In the first stage a segment of the isoelectric line is detected, in the time interval $[a-120 \mathrm{~ms}, a]$, where $a$ is the lower amplitude of QRS complex before the $\mathrm{R}$ wave. The segment is found if eight successive differences between neighbouring samples are smaller than a predefined value $c$ and the difference between the initial and final sample are also smaller than $c$ :

$c=0.01(\max Q R S-\min Q R S)$,

where $\max Q R S$ and $\min Q R S$ are the maximum and the minimum amplitude of the QRS complex, respectively. The start point of the first search interval is the first point of the isoelectric line segment. The end point of the first search interval is determined if a peak or a slope, what appears first, is detected. A peak is detected if, for a given sample $S_{i}$, the differences $S_{i}-S_{1}$ and $S_{i}-S_{2}$ have the same sign and are larger than $c . S_{1}$ is the point $10 \mathrm{~ms}$ before $S_{i}$ and $S_{2}$ is the point $10 \mathrm{~ms}$ after the $S_{i}$. A slope is detected if eight successive differences between points with $5 \mathrm{~ms}$ intervals have the same sign and are smaller than $1.5 \mathrm{c}$. The midpoint of the slope or the peak is considered as the end point of the first search interval. Using the same method with different $c$, the second search interval (where the QRS end will be detected) is determined. The start of the QRS complex is detected in the first search interval as follows: for each sample $S_{i}$ belonging to the first search interval, the $S_{1} \hat{S}_{i} S_{2}$ angle is calculated. The start of the QRS complex is considered as the point, which corresponds to the minimum angle.

The QRS start and end points are used to define the ECG segments, which are the ECG signals between two consecutive QRS complexes, starting at the end point of the current QRS complex and ending at the start point of the next QRS complex (Fig. 1).

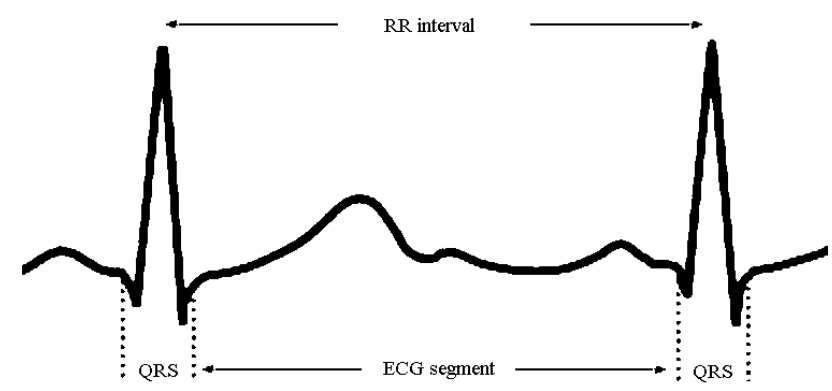

Figure 1. ECG segment.

\subsection{Time-frequency analysis and feature extraction}

The ECG segments are processed using timefrequency analysis. Smoothed Pseudo Wigner-Ville distribution (SPWVD) is used:

$$
\begin{aligned}
& \operatorname{SPWVD}(t, v)= \\
& \int_{-\infty}^{+\infty} h(\tau) \int_{-\infty}^{+\infty} g(s-t) x\left(s+\frac{\tau}{2}\right) x^{*}\left(s-\frac{\tau}{2}\right) d s e^{-j 2 \pi v \tau} d \tau,
\end{aligned}
$$

where $x$ is the signal, $x^{*}$ is its complex conjugate, $t$ is the time, $h(\cdot)$ is a frequency smoothing window and $g(\cdot)$ is a time smoothing window. Both, time and 
frequency smoothing windows were set as Hamming 63point length windows. The power spectrum density (PSD), which represents the fractional energy of the signal in time $t$ and frequency $f$, is computed (Fig. 1a). A grid of 40 squares is defined, selecting 5 equally spaced points for time and 8 equally spaced points on the frequency axis (fig 1b). The energy for each defined square of the grid is computed and the result is a vector of energies of dimension 40. This vector is the input to the neural network.


Figure 2. (a) PSD for an ECG segment; (b) 40 square grid.

\subsection{Atrial tachyarrhythmias classification}

Atrial tachyarrhythias classification is performed using a feed-forward back-propagation neural network. The training and testing sets are shown in Table 2. Several neural network architectures have been tried and the one that performs better has been chosen: 40 inputs, 1 hidden layer with 20 neurons and 3 outputs, being real numbers between 0 and 1 . The classified category is defined with the maximum output. The training of the neural network ends when the square error is less than 0.01 or the training epochs are more than 2000.

Table 2. Training and test set.

\begin{tabular}{lcc}
\hline Cardiac rhythm & $\begin{array}{c}\text { \# of beats in } \\
\text { the training set }\end{array}$ & $\begin{array}{c}\text { \# of beats in } \\
\text { the test set }\end{array}$ \\
\hline Normal & 1,000 & 58,500 \\
Atrial Fibrillation & 1,000 & 39,000 \\
Atrial Flutter & 100 & 400 \\
\hline Total & 2,100 & 97,900 \\
\hline
\end{tabular}

\section{Results}

The proposed method is evaluated using the above described dataset. The results are shown in Tables 3 and 4. In Table 3 each element $x_{i j}$ represents the number of beats belonging to category $j$ and classified in category $i$. The sensitivity, specificity and positive predictive value are given in Table 4.

Table 3. Results of the proposed method.

\begin{tabular}{|c|c|c|c|c|}
\hline & & \multicolumn{3}{|c|}{ Dataset } \\
\hline & & Normal & $\begin{array}{c}\text { Atrial } \\
\text { Fibrillation }\end{array}$ & $\begin{array}{l}\text { Atrial } \\
\text { Flutter }\end{array}$ \\
\hline \multirow{4}{*}{ 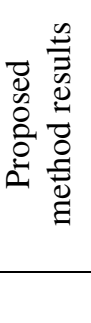 } & Normal & 55,378 & 1,924 & 9 \\
\hline & $\begin{array}{c}\text { Atrial } \\
\text { Fibrillation }\end{array}$ & 2,607 & 36,479 & 23 \\
\hline & $\begin{array}{l}\text { Atrial } \\
\text { Flutter }\end{array}$ & 515 & 597 & 368 \\
\hline & Total & 58,500 & 39,000 & 400 \\
\hline
\end{tabular}

Table 4. Sensitivity, specificity and positive predictive valus results.

\begin{tabular}{lccc}
\hline Cardiac rhythm & $\begin{array}{c}\text { Sensitivity } \\
\%\end{array}$ & $\begin{array}{c}\text { Specificity } \\
\%\end{array}$ & $\begin{array}{c}\text { Positive } \\
\text { predictive } \\
\text { value \% }\end{array}$ \\
\hline Normal & 94.66 & 95.09 & 96.63 \\
Atrial & 93.54 & 95.53 & 93.28 \\
$\begin{array}{l}\text { Fibrillation } \\
\text { Atrial Flutter }\end{array}$ & 92.00 & 98.86 & 24.86 \\
\hline
\end{tabular}

The accuracy of the proposed method is $94.20 \%$.

\section{Discussion and conclusions}

A method for automated classification of atrial tachyarrhythmias in ECGs is proposed, combining timefrequency analysis and ANNs The results indicate high performance in atrial flutter and atrial fibrillation beat classification. Results for sensitivity and specificity are high for all categories (92\% - 99\%). Positive predictive results are high for NSR and AFIB categories (96\% and 93\% respectively) but not for AFL (24\%). This is due to the small number of AFL beats (400), compared to the number of beats used for the other categories $(58,500$ for NSR and 39,000 for AFIB) in the dataset. 
Table 5. Comparison with other methods proposed in the literature.

\begin{tabular}{|c|c|c|c|c|c|c|}
\hline \multirow[b]{2}{*}{ Authors } & \multirow[b]{2}{*}{ Method } & \multirow[b]{2}{*}{ Database } & \multirow[b]{2}{*}{ Arrhythmic Types } & \multicolumn{3}{|c|}{ Results } \\
\hline & & & & $\begin{array}{c}\text { Sensitivity } \\
\%\end{array}$ & $\begin{array}{c}\text { Specificity } \\
\%\end{array}$ & $\begin{array}{c}\text { Accuracy } \\
\%\end{array}$ \\
\hline Yang et al. [1] & $\begin{array}{c}\text { Neural } \\
\text { networks }\end{array}$ & $\begin{array}{c}2363 \text { beats } \\
\text { Glasgow } \\
\text { Royal Infirmary }\end{array}$ & $\begin{array}{l}\text { Atrial fibrillation } \\
\text { Normal }\end{array}$ & 92 & 92.3 & \\
\hline Christov et al. [2] & $\begin{array}{c}\text { Rules } \\
\text { flowchart }\end{array}$ & $\begin{array}{c}329 \text { records } \\
(8 \text { sec length }) \\
\text { Sofia Medical Hospital }\end{array}$ & $\begin{array}{c}\text { Atrial } \\
\text { flutter/fibrillation } \\
\text { Normal }\end{array}$ & 95.7 & 98.3 & 98.8 \\
\hline Rieta et al. [3] & $\begin{array}{c}\text { Blind } \\
\text { source } \\
\text { separation } \\
\text { and } \\
\text { Fourier } \\
\text { analysis }\end{array}$ & $\begin{array}{c}30 \text { records } \\
(6 \text { sec length }) \\
\text { Hospital } \\
\text { Clinico de Valencia }\end{array}$ & $\begin{array}{c}\text { Atrial flutter } \\
\text { Atrial fibrillation } \\
\text { Normal }\end{array}$ & & & 100 \\
\hline this work & $\begin{array}{l}\text { Time- } \\
\text { frequency } \\
\text { analysis }\end{array}$ & $\begin{array}{c}100.000 \text { beats } \\
\text { MIT-BIH arrhythmia } \\
\text { and atrial fibrillation } \\
\text { databases }\end{array}$ & $\begin{array}{c}\text { Atrial flutter } \\
\text { Atrial fibrillation } \\
\text { Normal }\end{array}$ & $\begin{array}{l}94.66 \\
93.54 \\
92.00\end{array}$ & $\begin{array}{l}95.09 \\
95.53 \\
98.86\end{array}$ & 94.20 \\
\hline
\end{tabular}

Table 5 summarizes the results of other methods proposed in the literature. Some of the methods distinguish between only two categories (AFIB and SNR in [1], AFIB/ AFL and SNR in [2]). The datasets used [13] are much smaller $(300-6.000$ beats approximately) than the dataset used for the evaluation of the proposed method ( 97.900 beats). The results compare well with all other methods. Some of the methods [2] extract and use several features from ECG, such as the $\mathrm{P}$ wave, which is an extremely time-consuming process and sometimes difficult due to the presence of noise, especially in tachyarrhythmic cardiac episodes. This is avoided in our method, which makes our approach much faster.

\section{Acknowledgements}

This research was partially funded by the program "Heraklitos" of the Operational Program for Education and Initial Vocational Training of the Hellenic Ministry of Educationunder the 3rd Community Support Framework and the European Social Fund.

\section{References}

[1] Yang TF, Devine B, Macfarlane PW. Artificial neural networks for the diagnosis of atrial fibrillation. Med. \& Bio. Eng. \& Comput. 1994;32:615-9.
[2] Christov I, Bortolan G, Daskalov I. Sequential analysis for automatic detection of atrial fibrillation and flutter. In: Murray A. Computers in Cardiology 2001. Piscataway: IEEE, 2001:293-296.

[3] Rieta JJ, Millet-Roig J, Zarzoso V, Castells F, Sanchez C, Garcia-Civera R, Morell S. Atrial fibrillation, atrial flutter and normal sinus rhythm discrimination by means of blind source separation and spectral parameters extraction. In: Murray A. Computers in Cardiology 2002. Piscataway: IEEE, 2002:25-28.

[4] MIT-BIH Arrhythmia Database CD-ROM. Third Edition, 1997, Harvard-MIT Division of Health Sciences and Technology.

[5] MIT-BIH Atrial Fibrillation Database CD-ROM. Third Edition, 1997, Harvard-MIT Division of Health Sciences and Technology.

[6] Pan J, Thompkins WJ. A real-time QRS detection algorithm. IEEE Trans. Biom. Eng. 1985;32:230-6.

[7] Daskalov IK, Christov II. Electrocardiogram signal preprocessing for automatic detection of QRS boundaries. Med. Eng. \& Phys 1999;21:37-44.

Address for correspondence.

Dimitrios I. Fotiadis

Unit of Medical Technology and Intelligent Information Systems, Dept. of Computer Science, University of Ioannina Campus, P.O. BOX 1186, GR 45110 Ioannina, Greece.

fotiadis@cs.uoi.gr 